if the two-thirds law is obeyed. In an experiment carried out between 12.00 and 15.00 GMT on the 11 th July 1976, the mean ratio value was found to be 6.18 though the two logamplitude variances showed very good correlation. The highest ratio value obtained is 13.3, and the lowest 3.04. Inspection of the temperature spectra obtained during that period showed that the spectra slopes were fairly constant at approximately $-5 / 3$ but the outer scale size varied from $2.2 \mathrm{~m}$ to over $50 \mathrm{~m}$.

\section{CONCLUSIONS}

The results obtained from this ex periment show remarkably good agreement between experimental and theoretical values. It was found that the logarithmic amplitude variances at frequencies of $36 \mathrm{GHz}$ and $110 \mathrm{GHz}$ have a $k^{7 / 6}$ wavenumber dependence (the two-thirds law is assumed) when the condition $\sqrt{\lambda L}<L_{0}$ exists, and a $k^{2}$ wavenumber dependence when $\sqrt{\lambda L}>L_{0}$.

These results clearly demonstrate the importance of the effect of the size of the outer scale of turbulence on amplitude scintillations at millimeter wavelengths even when measurements are made when the "two-thirds law" holds. The applicability of the turbulence theory due to Tatarski at millimeter wavelengths is also clearly established.

\section{ACKNOWLEDGMENT}

Thanks are due to the Appleton Laboratories, which loaned the $110-\mathrm{GHz}$ equipment.

\section{REFERENCES}

[1] J. A. Lane, "Scintillation and absorption fading on line of sight

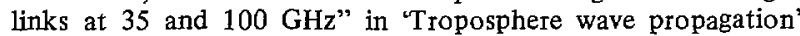
IEE Conf. Publ., 48., pp. 166-173, 1968.

[2] "Special Issue on Remote Environmental Sensing," in Proc. IEEE., vol. 57, pp. 371-742, Apr. 1969.

[3] R. W. Lee and A. T. Waterman, "Space correlation of $35 \mathrm{GHz}$ transmissions over a $28 \mathrm{~km}$ path," Radio Sci., vol. 3 , pp. $135-$ $140,1968$.

[4] K. L. Ho, N. D. Mavrokoukoulakis, and R. S. Cole, "Wavelength dependence of scintillation fading at 110 and $36 \mathrm{GHz}$," Electron. Lett., vol. 13, no. 7. pp. 181-183, 1977.

[5] S. M. Rytov, "Diffraction of light by ultrasonic waves," Izv. Akad. Nauk. SSSR Ser. Fiz, no. 2. p. 223, 1937.

[6] V. I. Tatarski, Wave Propagation in a Turbulent Medium. New York: Dover, 1961.

[7] S. F. Clifford and J. W. Strohbehn, "The theory of microwave line-of-sight propagation through a turbulent atmosphere," IEEE Trans. Antennas Propagat., vol. AP-18, no. 2, pp. 264-274, Mar. 1970.

[8] R. W. Lee and J. C. Harp, "Weak scattering in random media with application to remote probing," in Proc. IEEE, vol. 57 , no. 4, pp. 375-406, Apr. 1969.

[9] A. N. Kolmogorov, "The local structure of turbulence in incompressible viscous fluid for very large Reynolds' numbers," Doklady Akad. Nauk SSSR, 30, p. 301, 1941.

[10] J. S. Bendat and A. B. Piersol, Measurement and Analysis of Random Data. New York: Wiley, 1966.

[11] D. T. Gjessing, A. G. Kjelass, and E. Golton, "Small scale atmospheric structure deduced from measurements of temperature, humidity and refractive index," Boundary-Layer Meterol, pp. $475-492,1972$.

\title{
Fading at 9.6 GHz on an Experimentally Simulated Aircraft-to-Ground Path
}

\author{
H. B. JANES AND M. C. THOMPSON, JR.
}

\begin{abstract}
This report describes an experimental study of fading at 9.6 $\mathrm{GHz}$ on a fixed slanted line-of-sight path simulating aircraft-toground propagation conditions. Recordings of signal fading were made over about one year. The data were analyzed to provide a statistical description of the fading, and to compare some of these statistics with those obtained elsewhere under different climatic and terrain conditions. The results include the probabilities of occurrence of fading rates and fade durations at several signal levels below the hourly median level. The extent to which fading behavior depends on time-of-day and time-of-year was investigated, along with the correlation of hourly average fade duration and hourly fading rate.
\end{abstract}

\section{INTRODUCTION}

D URING January 1973 to January 1974, the Institute for Telecommunication Sciences made nearly continuous recordings of signal strength variations on a fixed line-of-sight 9.6-GHz link chosen to simulate low-angle aircraft-to-ground propagation conditions. The purpose was to obtain a data base

Manuscript received May 23, 1977; revised November 9, 1977. This work was supported by the Rome Air Development Center, USAF under Contract No. F 30602-75-F-0256.

The authors are with the Institute for Telecommunication Sciences, Office of Telecommunications, U.S. Department of Commerce, Boulder, CO 80302 . for estimating the probable frequency of occurrence of fades of various depths and also to estimate the statistics of fade duration.

The $120-\mathrm{km}$ propagation path extended from the summit of Mt. Haleakala on the Island of Maui, Hawaii, at an elevation of $3000 \mathrm{~m}$, to a point near the Keahole Airport on the Island of Hawaii, at an elevation of $20 \mathrm{~m}$. The horizontally polarized transmitting and receiving antennas were a 3-m diameter parabola (beam width: $0.8^{\circ}$ ) on Mt. Haleakala and a $2.4-\mathrm{m}$ diameter parabola (beam width: $0.9^{\circ}$ ) at the Keahole site. Since the angle of elevation of the path was about $1^{\circ}$, none of the Earth's surface was contained within the space illuminated by the main beams of both antennas. Hence, it is assumed that none of the observed fading was due to surface reflection interference.

\section{DATA PROCESSING}

The data consist of 147 1-h samples of signal strength variations selected from January 1973 to January 1974. The samples were taken from the hours 0000-0100, 0600-0700, 1200-1300, and 1800-1900 during one day per week (arbitrarily chosen as Wednesday, or the nearest day during which usable data were recorded). Data processing consisted of computing the cumulative distribution of signal level for each hour 


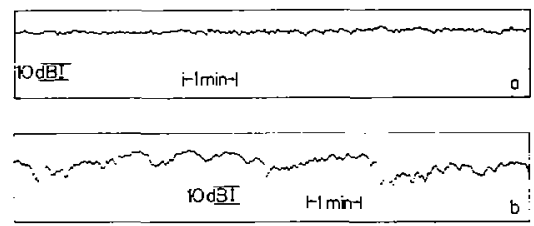

Fig. 1. Examples of $9.6 \mathrm{GHz}$ signal variations during periods of (a) relatively shallow and (b) relatively deep fading.

and then measuring the duration of each fade below each of several reference levels (i.e., 3, 5, 7, 10, 15, 20, 25, and $30 \mathrm{~dB}$ below the hourly median). The maximum fade depth was also measured for each hour, again in terms of decibels below the hourly median.

\section{RESULTS}

Two typical examples of signal strength variations are shown in Fig. 1, one taken from a relatively quiet period and one from a.period of active and occasionally deep fading. The cumulative distributions for the two hours from which these examples were taken are shown in Fig. 2. Since the vertical scale on Fig. 2 is arbitrary, the relative vertical positioning of the two distributions on the graph is not significant. Only the slopes of the distributions (in terms of the 1-99 percent fading ranges), the maximum fade depth, and the number and duration of fades below the several levels relative to the hourly median were used in this analysis.

\section{A. Fade Duration}

Figs. 3-7 summarize the statistics of the fade durations. Fig. 3 gives the cumulative distributions of durations of all fades below the indicated levels that occurred in the entire data base. For example, the top curve is the distribution of the durations of all of the 11788 fades that fell below the 3-dB level. Of those fades, only 6165 also fell below the $5 \mathrm{~dB}$ level, 3500 fell below $7 \mathrm{~dB}$, and so on. It can be seen that as the number of fades decreases, so does the statistical reliability of the distributions, and hence the decreased smoothness of the curves. The least count of the duration measuring process was $0.64 \mathrm{~s}$, and this constitutes the lower limit on all of the duration data. Fig. 4 shows the maximum and average duration observed for each reference level. Although the average duration only changed by a factor of about 5 between 3 and $30 \mathrm{~dB}$, the maximum duration changed by a factor of almost 1000 .

Figs. 5 and 6 show the duration data for the 3-dB and $15-$ $\mathrm{dB}$ fade levels, broken down into separate distributions for each of the four hours of the day. Although the shape of the distribution does not change significantly with time of day, there appears to be a tendency toward somewhat shorter fade durations during the hour 0000-0100 than during the other three hours.

In Fig. 7 , the average fade durations at the $3,5,7,10$, and 15-dB levels are plotted versus hour of the day. Here the tendency toward shorter fades during the hour 0000-0100 is even more obvious than in the distributions mentioned above. In addition, the diurnal variation pattern also appears to be a function of reference level. The maximum average duration occurs during 1800-1900 at the 3 and 5-dB levels, and during $0600-0700$ at the 7,10 , and 15 -dB levels.

To check for a possible seasonal variation in fade durations, the hourly average fade durations at the 3 and 10-dB levels were examined versus time of year, but showed no identifiable seasonal dependence.

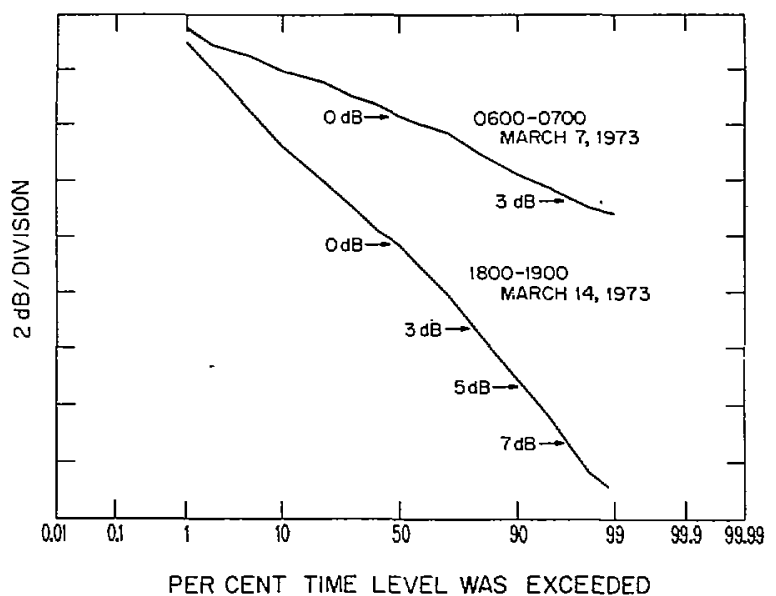

Fig. 2. Cumulative distribution of signal levels during periods represented in Fig. 3.

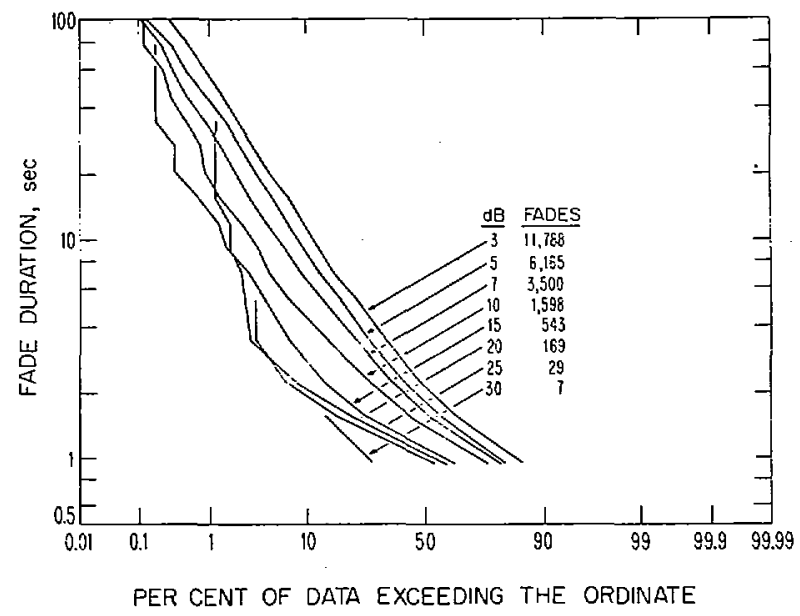

Fig. 3. Cumulative distributions of fade durations, $4 \mathrm{~h} /$ day, all months combined.

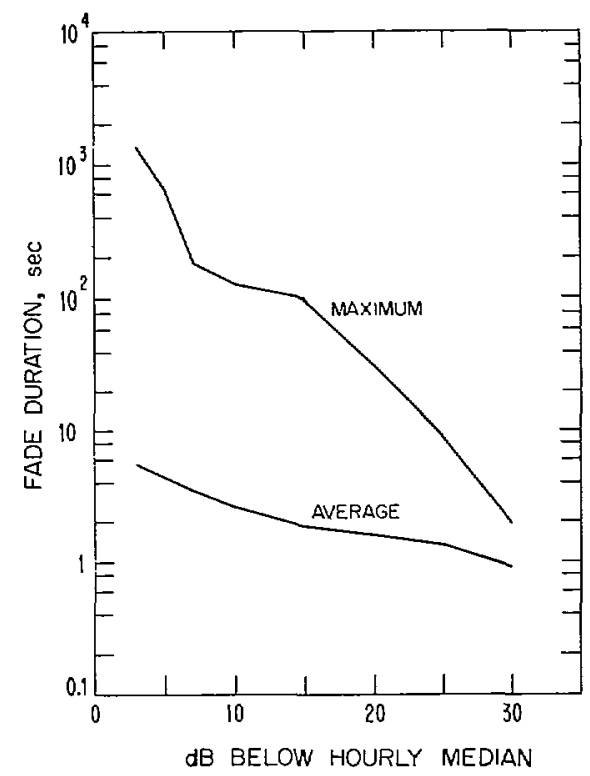

Fig. 4. Fade duration versus signal level, $4 \mathrm{~h} /$ day, all months combined. 


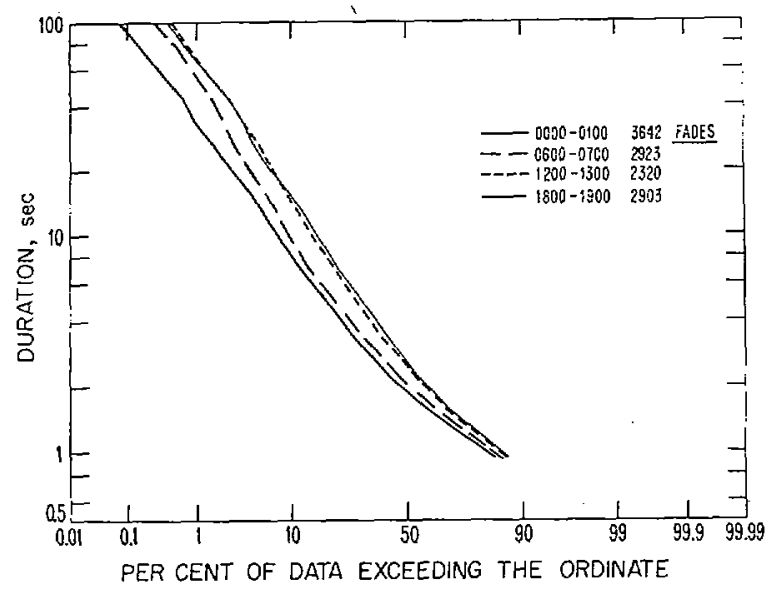

Fig. 5. Cumulative distributions of fade durations, all months combined, 3-dB level.

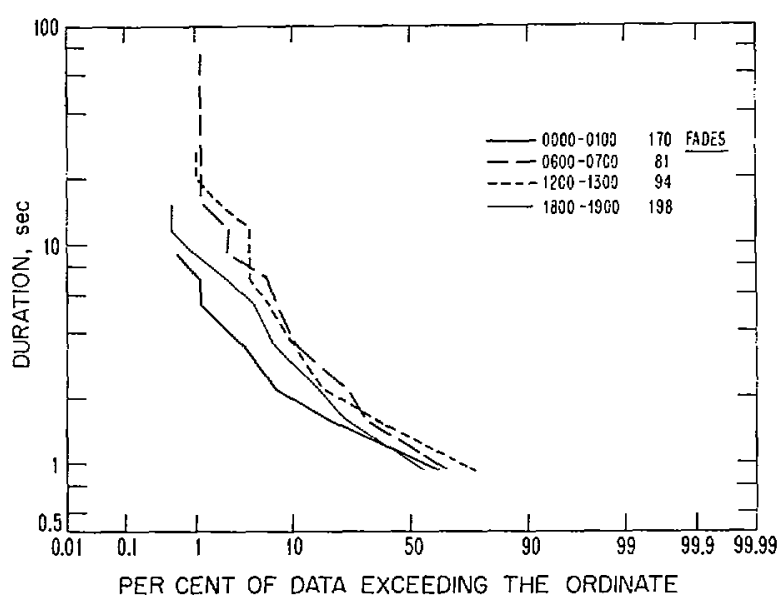

Fig. 6. Cumulative distributions of fade durations, all months combined, 15-dB level.

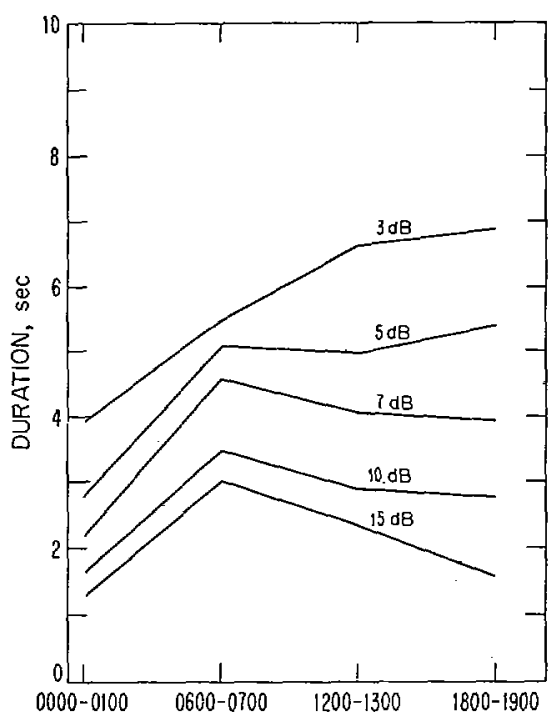

Fig. 7. Average fade duration versus time of day, all months combined.

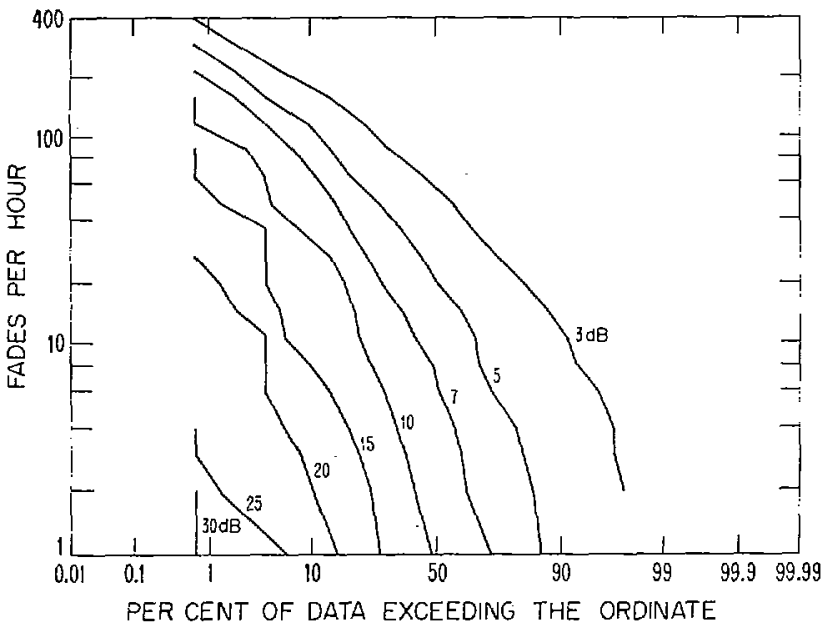

Fig. 8. Cumulative distribution of hourly fading rates, $4 \mathrm{~h} / \mathrm{day}$, all months combined.

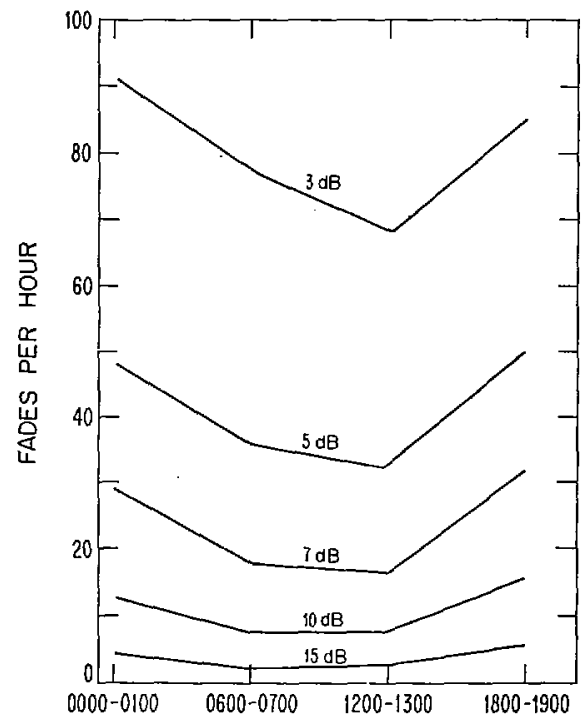

Fig. 9. Average fading rate versus time of day, all months combined.

\section{B. Fading Rates}

Figs. 8-10 summarize the statistics of the fading rate, defined here as the number of times during a given hour that the signal faded below a given reference level. Again, all reference signal levels are expressed in terms of decibels below the hourly median level. Fig. 8 shows cumulative distributions of the hourly fading rates for each of the several reference levels. Unlike the fade duration data which were nearly log-normal in distribution (Fig. 3 and Figs. 5 and 6), neither the fading rates nor their logarithms closely approximated the normal distribution. They are shown on a log-normal graph (Fig. 8) simply to provide good graphical resolution over the entire range of fading rates. In Fig. 9, the average fading rates for each of the four hours of the day at the $3,5,7,10,15$-dB levels are plotted versus time of day. In general, the fading rates tended to be lower during the daylight hours.

\section{Correlation of Fading Rates and Average Fade Durations}

One might expect intuitively that there would be a negative correlation between the number of fades during a given hour 


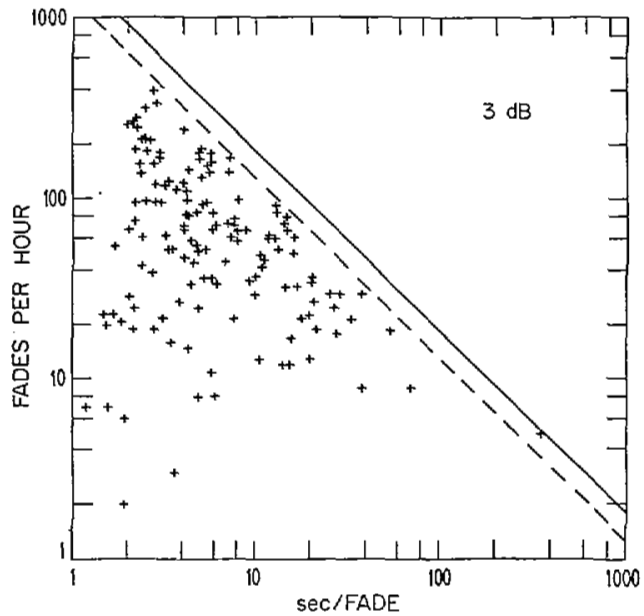

Fig. 10. Hourly fading rates versus average fade duration, $4 \mathrm{~h} /$ day, all months combined, 3-dB level. Solid line: expected upper bound. Dashed line: "observed" upper bound.

and the average duration of those fades. In other words, the higher the fade count, the shorter would be the average fade duration. An effort to establish the existence of such a correlation produced a very interesting result. In Fig. 10, the hourly fading rates are plotted versus the corresponding hourly average fade durations for the $3-\mathrm{dB}$ signal level. A solid diagonal line has been drawn representing a value of 1800 for the product of rate and average duration. All of the data would fall along this line if the fades had been measured at the hourly median instead of 3-dB below the median. Clearly, none of the 3-dB points could be above and to the right of the diagonal line (i.e., the product cannot be greater than $1800 \mathrm{~s}$ for any signal level below the median). However, it is interesting to note that (except for the inevitable "anomalous" point at the lower right!) all of the data appear to observe a "minimum distance" from the median line. The dashed line is an approximate upper limit to the data and corresponds to a value of $1200 \mathrm{~s}$ for the maximum observed product of rate and duration. This means that, in spite of the wide variation of fading rates and average fade durations, the signal was more than $3 \mathrm{~dB}$ below the hourly median no more than $1200 \mathrm{~s}$ or 33 percent of any hour. Aside from this apparent maximum permissible duration of the signal below the 3- $\mathrm{dB}$ level, the data points are scattered randomly, and there appears to be no significant correlation between fade rate and duration.

The approximate maximum values of the product of rate and duration for each of the levels were used to construct the "maximum" cumulative signal distribution in Fig. 11. This curve represents the maximum percentage of time that the signal was below the indicated reference levels. For comparison, the average cumulative distribution is also shown.

\section{1-99 Percent Fading Ranges}

The 1-99 percent fading range is defined as the difference in decibels between the signal levels exceeded 1 percent and 99 percent of the time during a given hour. These data are summarized in Fig. 12 by means of cumulative distributions of hourly fading ranges observed during each of the four hours of the day. Although the extreme high values occurred during the hours $0600-0700$ and $1200-1300$, the average fading range was highest during the hour 1800-1900.

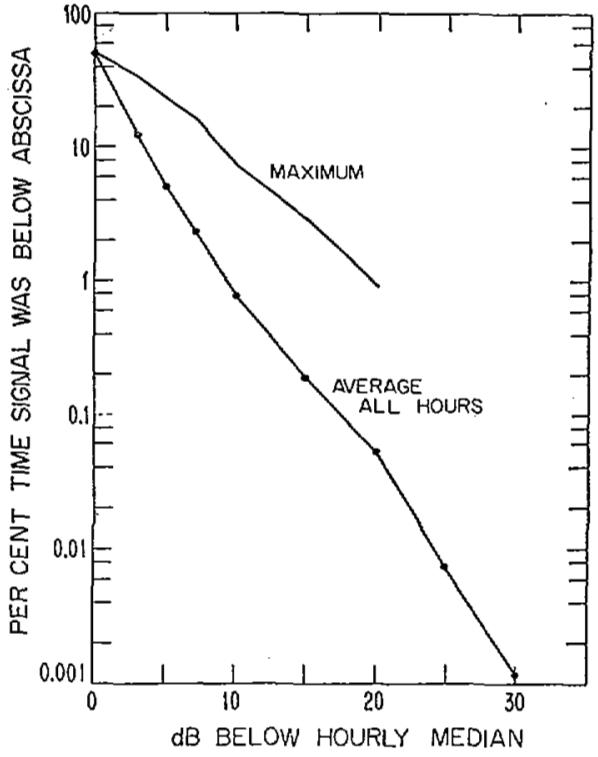

Fig. 11. Maximum and average cumulative distributions of signal level relative to hourly median, all months combined.

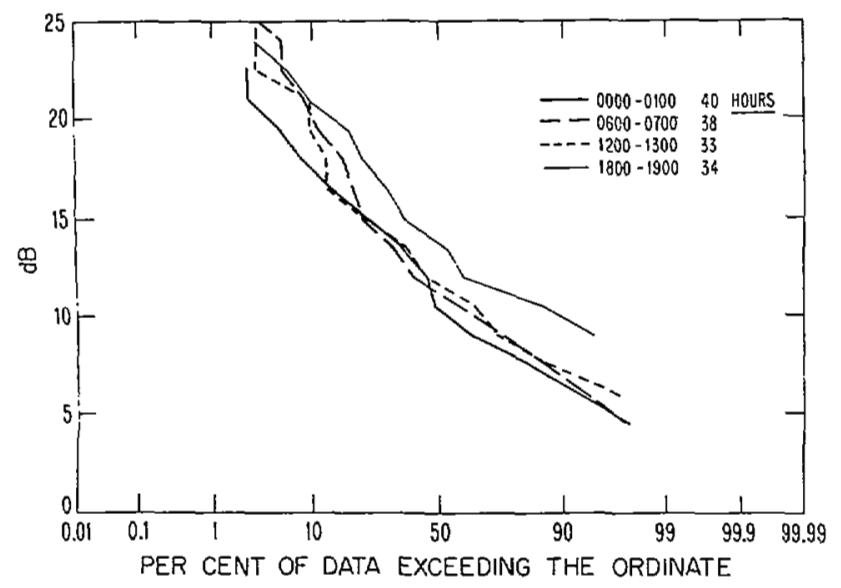

Fig. 12. Cumulative distributions of hourly 1-99 percent fading ranges, all months combined.

The maximum value of the 1-99 percent fading ranges was $28 \mathrm{~dB}$, about 5 percent of the values were larger than $22 \mathrm{~dB}$, and the median fading range for all hours was about $12 \mathrm{~dB}$.

\section{COMPARISON WITH OTHER RESULTS}

A remaining question has to do with the "portability" of these results, i.e., the extent to which data taken on an overwater propagation path in Hawaii resembles the results that would be obtained on propagation paths over different terrain and in different climates. A partial answer to this question can be obtained by comparing these results with those obtained by W. T. Barnett [1] from an experiment in Ohio. In his experiment, an 11-GHz signal was transmitted over a $46-\mathrm{km}$ overland path between antennas 77 and $97 \mathrm{~m}$ above ground. Ground reflections were considered to be negligible. Fading data were recorded during the period July 22-September 28, 1966. Fade depths were given in terms of $\mathrm{dB}=20 \log L$, where $L$ is the "normalized algebraic value of envelope voltage" relative to 


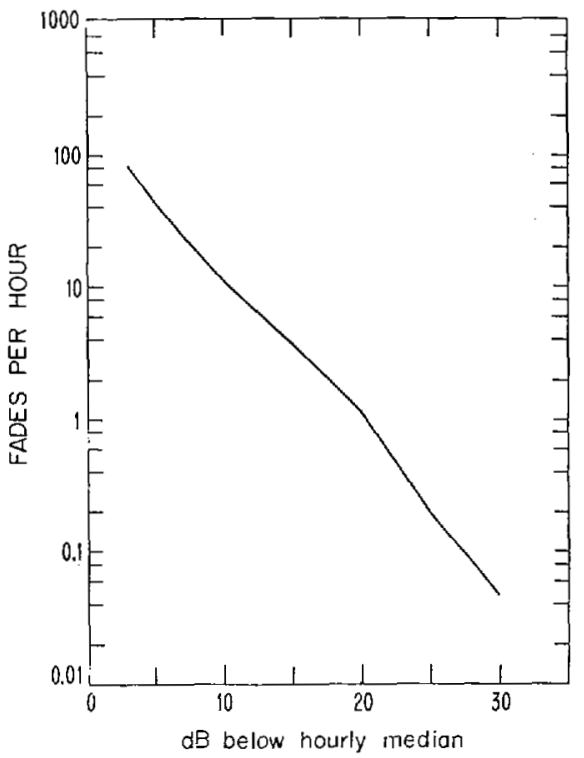

Fig. 13. Average fading rate versus fading level, all months and hours combined.

the "mid-day normal" signal level. Barnett found that, in the cumulative distribution of fade depths (his Fig. 3), the fraction of time that the fade depth equaled or exceeded a given level, $L$, was proportional to $L^{2}$. Fig. 11 in this report indicates that the fraction of time is proportional to $L^{3.2}$.

Again, Barnett found that the total number of fades below a given level $L$ was proportional to $L$ (although inspection of his Fig. 4 might lead one to favor an exponent of $L$ of less than unity). The corresponding average fade rate data in Fig. 13 of this report indicates that fading rate is proportional to $L^{2.4}$.

Barnett's data on average fade duration were found to be proportional to $L$, (see his Fig. 5), while those from the present experiment (Fig. 4) are approximately proportional to $L^{0.6}$. Since the fade depths in the present study are referred to the individual hourly medians instead of to a fixed long-term reference level, some differences in fading statistics are to be expected.

Good agreement was found in one statistic used by Barnett which was found to be independent of signal level, and which 7 was therefore unaffected by the choice of reference signal level. That statistic is the cumulative distribution of fade durations at a given signal level, normalized to the average duration for that level (see his Fig. 6). The corresponding distribution of

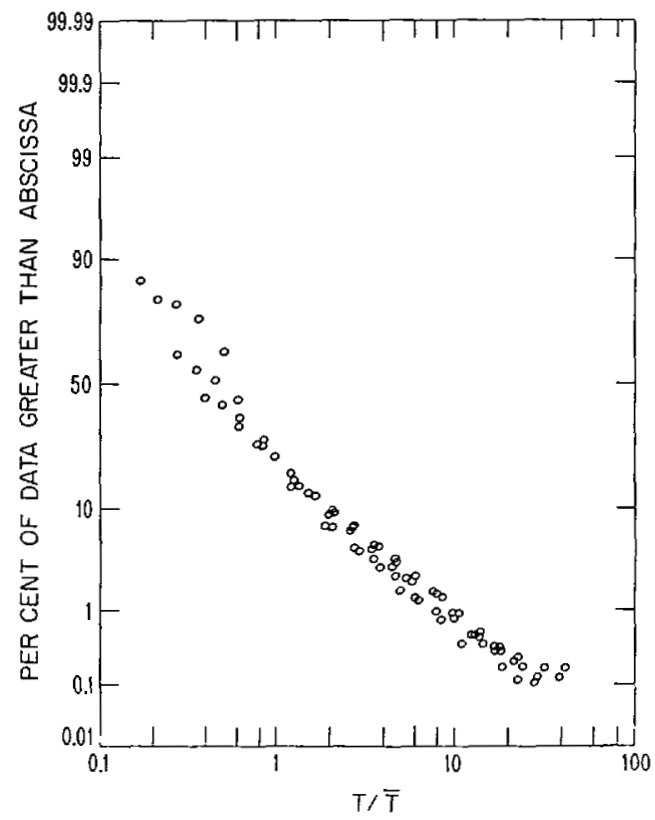

Fig. 14. Cumulative distribution of fade duration $\bar{T}$ normalized to average duration at given signal level $\bar{T}$; all months and hours combined; $3,5,7,10,15-\mathrm{dB}$ signal levels combined.

normalized fade durations for the present experiment is shown in Fig. 14. The latter agrees very well with Barnett's finding, i.e., that the normalized duration is independent of signal level, "and that the probability is approximately log normal, with 1 percent of the fades being longer than ten times the average fade duration."

This agreement between fade duration distributions from such widely-differing propagation paths as these is very encouraging evidence of the possible applicability of these results to other locations. The differences noted above may be the result of unavoidable differences in the choices of reference signal levels. They illustrate the need for further experimental studies of fading in which identical recording techniques at the various locations would permit identical data analysis methods. Only then can geographic and climatic effects on microwave fading be systematically investigated.

\section{REFERENCES}

[1] W. T. Barnett, "Multipath propagation at 4,6 , and $11 \mathrm{GHz}$," Bell Syst. Tech. J., vol. 51, no. 2, pp. 321-361, 1972. 